\title{
Radiating electron source generation in ultraintense laser-foil interactions
}

\author{
R. Capdessus, ${ }^{*}$ M. King, and P. McKenna ${ }^{\dagger}$ \\ Department of Physics SUPA, University of Strathclyde, Glasgow G4 ONG, UK
}

\begin{abstract}
A radiating electron source is shown to be created by a laser pulse (with intensity of $10^{23} \mathrm{~W} / \mathrm{cm}^{2}$ and duration equal to $30 \mathrm{fs}$ ) interacting with a near-critical density plasma. It is shown that the back radiation reaction resulting from high energy synchrotron radiation tends to counteract the action of the ponderomotive force. This enhances the collective dynamics of the radiating electrons in the highest field areas, resulting in the production of a compact radiation source (containing $80 \%$ of the synchrotron radiation emission), with an energy on the order of tens of $\mathrm{MeV}$ over the laser pulse duration. These phenomena are investigated using a QED-particle-in-cell code, and compared with a kinetic model accounting for the radiation reaction force in the electron distribution function. The results shed new light on electron-photon sources at ultra-high laser intensities and could be tested on future laser facilities.
\end{abstract}

\section{INTRODUCTION}

The emergence of laser facilities providing pulses with peak intensity exceeding $10^{23} \mathrm{~W} / \mathrm{cm}^{2}$ in the near future drives theoretical interest in the resulting new regimes which will be accessed experimentally. The APOLLON and ELI laser facilities will enable the so-called "ultrarelativistic regime' to be reached, in which charged particle dynamics are expected to be highly relativistic and can be affected by the radiation reaction (RR) force. This enables the exploration of novel matter properties in which collective charged-particle effects are important.

In laser-plasma interactions at intensities beyond $10^{22}$ $\mathrm{W} / \mathrm{cm}^{2}$, the backward reaction of the high-energy radiation generated by radiating electrons can strongly impact the overall plasma dynamics. The RR force generally acts to cool the electron dynamics [1-8], although it can enhance electron energy in some situations [9-12]. Green and Harvey [12] deduce that electron interactions with a strong laser pulse results in discrete photon emission, which introduces a transverse electron beam spread that is distinct from that due to classical effects. In addition, at such ultra-high laser intensities, the collective effects driven mainly by the charge separation field play a significant role in the plasma dynamics, in particular on the production of the high energy synchrotron radiation in the case of the hole boring regime where the target thickness is much larger than the laser wavelength [1316]. The collective dynamics of a longitudinal electron beam (i.e. where only the longitudinal component of the electron beam momentum is considered) has been investigated in references [17, 18], and the RR effect on the longitudinal electron distribution function deduced. RR is found to alter the beam dynamics. Quantum stochastic effects tend to induce the opposite effect to the classical $\mathrm{RR}$. Whilst classical RR reduces the energy spread of the electron beam, the quantum effects tends to increase the energy spread. This opposite behavior is due to the stochasticity of photon emission, which becomes dominant in the full quantum regime, at the expense of the incoherent emission of multiple photons in the classical regime. These results have been confirmed by a similar study in reference [19].

In this article, we demonstrate the prominent impact of the $\mathrm{RR}$ effect on the collective dynamics of radiating electrons, which tends to contain them in the highest field areas (in opposition to the ponderomotive force which expels them) during the interaction of an ultraintense laser pulse with a deuterium target. This can produce a compact high energy synchrotron radiation source of the order of tens of $\mathrm{MeV}$ over the laser pulse duration. We introduce an analytical model based on a kinetic approach, which confirms the main characteristics of the radiating electron source that are observed in ParticleIn-Cell (PIC) simulations. This builds upon the previous studies of the RR effect on electron beams during the interactions with a laser pulse [17-19], by extending to a laser-plasma interaction.

The remainder of the paper is organized as follows. Section II presents the electron equation of motion and a brief analysis of the high energy synchrotron radiation. Section III presents the numerical approach as well as the laser and plasma parameters considered. In Section IV, we describe two-dimensional (2D) PIC simulations of an ultraintense laser pulse interacting with a thin nearcritical density plasma, which demonstrates the formation of a radiating electron source. The characteristics of this electron population are then investigated. In Section $\mathrm{V}$, a kinetic model is derived and shown to accurately predict the characteristics of the radiating electron source. Finally, in section VI, we discuss and conclud the main results obtained.

\section{UNDERPINNING THEORY}

\section{A. Electron motion equation}

We define the dimensionless laser amplitude:

$$
a_{L}:=\frac{e E_{L}}{m c \omega_{L}} \gg 1,
$$


and the radiation parameter:

$$
R:=\omega_{L} \tau_{r} a_{L}^{2} \rho_{0}<1
$$

which marks the onset of the strong $\mathrm{RR}$ regime $[3,20]$. Here, $m$ is the electron mass, $\omega_{L}$ is the laser frequency, -e is the electron charge, $\tau_{r}=e^{2} / 6 \pi \epsilon_{0} m c^{3} \simeq 6.2 \times 10^{-24} \mathrm{~S}$ is the characteristic radiation time for an electron and $\rho_{0}$ the Doppler factor without any RR effect:

$$
\rho_{0}=\gamma(1-\boldsymbol{\beta} . \mathbf{n})
$$

where,

$\mathbf{n}=\frac{\mathbf{k}_{\mathbf{L}}}{k_{L}}, \gamma=\left(1-\boldsymbol{\beta}^{2}\right)^{-1 / 2}$ and $\beta=\frac{v}{c}$ are the normalized wavenumber of the laser field, the electron relativistic factor and the electron velocity normalized to the speed of light, respectively. To enable a strong interaction between laser field and electrons, and thus a significant photon emission, the following condition must be fulfilled:

$$
\frac{n_{e}}{n_{c}} \ll a_{L},
$$

where $n_{e}$ is the electron density, $n_{c}=m_{e} \epsilon_{0} \omega_{L}^{2} / e^{2}$ is the critical density and $\omega_{L}$ is the laser frequency. This corresponds to the relativistic self-induced transparency regime. Since $a_{L}$ is of the order of $10^{2}$ in the domain of interest, the parameter $R(2)$ is close to unity, which means that the electron dynamics can be modeled using a continuous approach, where the RR can be formalized by a friction force, by the Landau-Lifshitz equation [21]:

$$
\begin{array}{r}
\frac{d u^{a}}{d s}=-\frac{e}{m}\left(F^{a}{ }_{b}+\tau_{r} u^{c} \partial_{c} F^{a}{ }_{b}\right) u^{b} \\
+\tau_{r} \frac{e^{2}}{m^{2}} \Delta^{a}{ }_{b} F^{b}{ }_{c} F^{c}{ }_{d} u^{d},
\end{array}
$$

where $u^{a}=d r^{a} / d s=c \gamma(1, \boldsymbol{\beta})$ is the four electron velocity, $s$ is the electron proper time, $F^{a}{ }_{b}$ are components of the electromagnetic field tensor, and $\Delta^{a}{ }_{b}:=\delta_{b}^{a}-u^{a} u_{b} / c^{2}$ is the $\mathrm{u}$-orthogonal projection. Indices are raised and lowered with the metric tensor $\eta_{a b}=\operatorname{diag}(1,-1,-1,-1)$ and the Einstein summation convention is used.

To insure a semi-classical framework, the dimensionless parameter $\chi_{e}$ :

$$
\chi_{e}:=\frac{\sqrt{-\left[F^{a}{ }_{b} u^{b}\right]^{2}}}{E_{s}},
$$

which measures the significance of quantum effects, must be much less than unity. Here, $E_{s}=m c^{2} / e \lambda_{c}$ is the Schwinger field [22] with $\lambda_{c}=\hbar / m c$ the reduced Compton length. For $\chi_{e} \gtrsim 0.5$ the stochastic nature of photon emission becomes important, meaning that a radiating electron emits fewer photons per emission, compared to the classical radiation dominated regime [17, 23, 24]. For $\chi_{e} \sim 1$, the quantum nature of the radiation, with emitted photons having energy comparable to the electron energy, leads to discontinuous particle trajectories with significant recoils and non-negligible electron-positron pair creation [25-29]. In the semi-classical regime $\chi_{e} \sim 0.1$ a continuous treatment of the radiation emission is still possible, although the expressions for the total radiated power and energy spectra need to be corrected $[25,26,30]$.

\section{B. The synchrotron radiation}

In the semi-classical framework, the spectral intensity of the high energy synchrotron-like radiation emitted by an ultra-relativistic electron is given by [31]:

$$
\frac{d I_{r}}{d \omega d \boldsymbol{\Omega}}=P_{c} \boldsymbol{\delta}\left(\boldsymbol{\Omega}-\frac{\mathbf{u}}{\|\mathbf{u}\|}\right) S\left(\frac{\omega}{\omega_{c r}}\right),
$$

where $\mathbf{u}=c \gamma \boldsymbol{\beta}$. Here, $P_{c}=P g\left(\chi_{e}\right)$ is the instantaneous radiated power with $P=\tau_{r} \omega_{L}^{2} m c^{2} \chi_{e}^{2}$ the classical radiated power and $g\left(\chi_{e}\right) \approx$ $\left[1+4.8\left(1+\chi_{e}\right) \ln \left(1+1.7 \chi_{e}\right)+2.44 \chi_{e}^{2}\right]^{-2 / 3}$ the function accounting for the first quantum effects [37]. Even for $\chi_{e}=0.1$, the radiated power $P_{c}$ is reduced by $33 \%(g(0.1) \simeq 0.67)$ compared to its classical value. $S(r)=3^{5 / 2}(8 \pi)^{-1} r \int_{r}^{\infty} K_{5 / 3}\left(v^{\prime}\right) d v^{\prime}$ describes the normalized spectral shape with $\omega_{c r}=3 / 2 \gamma^{3}\left\|\mathbf{u} \times \mathbf{F}_{L}\right\| / m \mathbf{u}^{2}$. The radiation is emitted in a narrow cone with the angle $1 / \gamma$ with respect to the electron propagation direction and can be modeled by a Dirac function. Since the total radiation energy is calculated incoherently as a sum of all electrons, the corresponding wavelengths of photons are much smaller than the characteristic distance between electrons, $d \sim n_{e}^{-1 / 3}$, so they cannot be coherently summarized.

\section{NUMERICAL APPROACH}

\section{A. Numerical model}

In order to investigate collective and non-linear phenomena involving ultra-strong electromagnetic fields, 2D numerical simulations were performed using the QEDPIC code EPOCH [32]. It uses the Vlasov-Maxwell system with the inclusion of quantum processes such as the synchrotron gamma-ray photon emission from radiating electrons into the electromagnetic fields and pair production [30, 33-35]. The particles of the plasma (electrons, positrons and ions) are described by the quasi-classical model of Baier and Katkov [36] which means that the particles experience the Lorentz force and photon emission is computed with an emission probability [34, 35], inducing the lost electron momentum. The validity of having 
a semi-classical approach to describe the electron-photon dynamics has been discussed in references $[24,37]$ and confirmed in reference [38]. This implies that the plasma is transparent to high energy synchrotron radiation, due to the fact that the absorption rate of such radiation depends on the quantum parameter $\chi_{e}$. The absorption rate becomes non-negligible for electrons having $\chi_{e} \geq 1$ $[34,35]$. As a result, $e^{-} e^{+}$pair production is not considered.

\section{B. Simulation parameters}

The laser pulse is considered to be circularly polarized in order to optimize the collective effects whilst avoiding strong electron heating. The pulse is normally incident on a deuteron plasma slab with a thickness of $0.8 \mu \mathrm{m}$ and an initial electron density $\left(n_{e}\right)$ of $10 n_{c}$. Here, $a_{L}=$ $a_{y}=a_{z}=200$ which corresponds to a laser intensity of $1.1 \times 10^{23} \mathrm{~W} / \mathrm{cm}^{2}$ and ensures condition (4) is satisfied. The spatial and temporal structure of the laser is defined to be Gaussian in profile. The duration $\left(\tau_{L}\right)$ is $9.1 T_{L} \simeq$ $30 \mathrm{fs}$ with a beam-waist $\left(w_{0}\right)$ of $15 \lambda_{L}$ where $\lambda_{L}=1 \mu \mathrm{m}$ is the laser wavelength.

The laser pulse begins to interact with the plasma foil at $t=0$ and propagates along the $x$ axis $\left(\mathbf{n} \equiv \mathbf{e}_{x}\right)$. We thus define the polar angle $\theta$ as follows:

$$
\theta:=\left\{\begin{array}{l}
\arctan \left(\frac{\beta_{y}}{\beta_{x}}\right), \text { if } \beta_{x}>0 \\
\arctan \left(\frac{\beta_{y}}{\beta_{x}}\right)+\pi, \text { if } \beta_{x}<0 \text { and } \beta_{y} \geq 0 \\
\arctan \left(\frac{\beta_{y}}{\beta_{x}}\right)-\pi, \text { if } \beta_{x}<0 \text { and } \beta_{y}<0 \\
\frac{\pi}{2}, \text { if } \beta_{x}=0 \text { and } \beta_{y}>0 \\
-\frac{\pi}{2}, \text { if } \beta_{x}=0 \text { and } \beta_{y}<0
\end{array}\right.
$$

The simulation box is defined by a spatial grid of dimensions $200 \mu \mathrm{m} \times 57.6 \mu \mathrm{m}$ using $20000 \times 5760$ mesh cells. Since circular polarization is considered, there is symmetry between the $\mathrm{X}-\mathrm{Y}$ and $\mathrm{X}-\mathrm{Z}$ planes. Hence, the third spatial dimension does not have a strong impact on the radiation reaction physics highlighted in the remainder of the paper. 2D numerical simulations are therefore adequate for this study.

\section{NUMERICAL SIMULATIONS RESULTS}

\section{A. Formation of the radiating electron source}

As the laser pulse interacts with the target, a return current is generated, due to charge conservation, at $t \sim 3 T_{L}$ and interacts strongly with the rising edge of the laser pulse. We stress that the ions are not yet in motion at this early time, and do not affect the electron return current. These electrons thus experience the envelope of the laser pulse and acquire large energies as

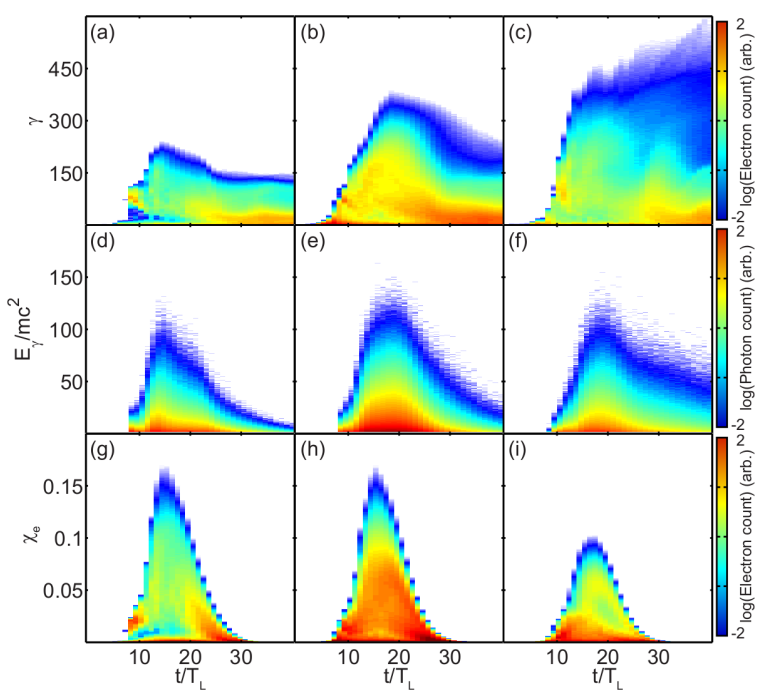

Figure 1: (Color online) (a)-(c) Electron spectra and (d)-(f) Radiated power, and $(\mathrm{g})-(\mathrm{i}) \chi_{e}$ parameter as a function of time. (a),(d),(g): $3 \pi / 4 \leq|\theta| \leq \pi ;(\mathrm{b}),(\mathrm{e}),(\mathrm{h}): \pi / 4 \leq|\theta| \leq 3 \pi / 4$;

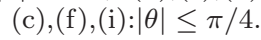

shown in figure $1(\mathrm{~b})$. At $t \gtrsim 14 T_{L}$, the $\mathrm{RR}$ force has a significant influence on this electron population, due to the fact that the laser intensity now exceeds $\approx 5 \times 10^{22}$ $\mathrm{W} / \mathrm{cm}^{2}$. In particular, the electrons which propagate toward the laser pulse reach their maximum energy $\approx 136$ $\mathrm{MeV}$, as can be seen in figure 1(a). They lose the bulk of their kinetic energy and a significant number of them are reflected by the laser pulse, acting as a mirror.

To be reflected by the laser pulse the following condition must be fulfilled $[3,4]$ :

$$
\begin{aligned}
& R \geq \frac{4 \gamma^{2}(t)-a_{L}^{2}(t)}{2 a_{L}^{2}(t)}>0, \\
& \Rightarrow \gamma(t) \geq \frac{a_{L}(t)}{2},
\end{aligned}
$$

which is in good agreement with the features in figure $1(\mathrm{a})$.

The electrons therefore experience areas of high field strength for a longer time giving rise to further RR effects and increased production of high energy synchrotron radiation.

$\mathrm{RR}$ force tends to both decrease the maximum energy of backward-directed electrons by $\sim 20 \%$ and increase the maximum energy of forward-directed electrons by $\sim 21 \%$ as shown in figure 2. This also tends to enhance the number of electrons experiencing the action of the laser pulse, such as $\pi / 4 \leq|\theta| \leq 3 \pi / 4$, as shown by the dense lobes in figure 3 . In the remainder of the paper, this electron population will be termed the radiating electron source $(\mathrm{RES})$, created at $t_{0} \approx 15 T_{L}$. 


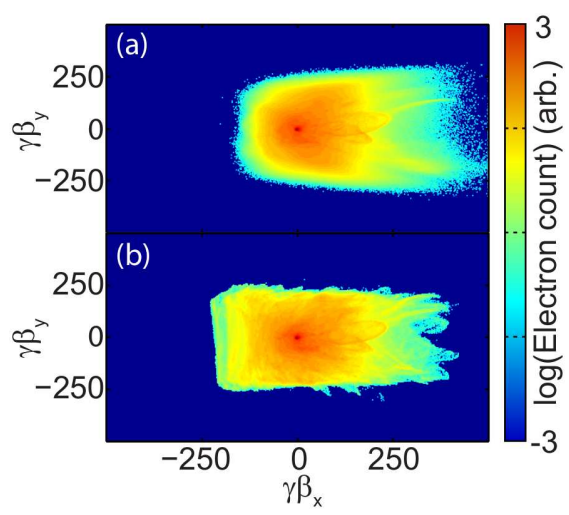

Figure 2: (Color online) Electron distribution function $u_{y}=f\left(u_{x}\right)$ at $t=18 T_{L}$ : (a) with $\mathrm{RR}$ and (b) without RR.

\section{B. Features of the radiating electron source}

In order to demonstrate the generation of the RES due to the counteracting action of the $\mathrm{RR}$ force on the action of the ponderomotive force, the angular distribution of the RES during the laser pulse duration is shown in figure 3. The

electrons considered are contained within a transverse window of width $3 \lambda_{L}$, centered on the laser propagation axis $(y=0)$. This implies $\partial a_{L} / \partial y \sim 0$ for $-1.5 \lambda_{L} \leq$ $y \leq 1.5 \lambda_{L}$, owing to the value of the laser focal spot size $\left(w_{0}=15 \lambda_{L}\right)$.
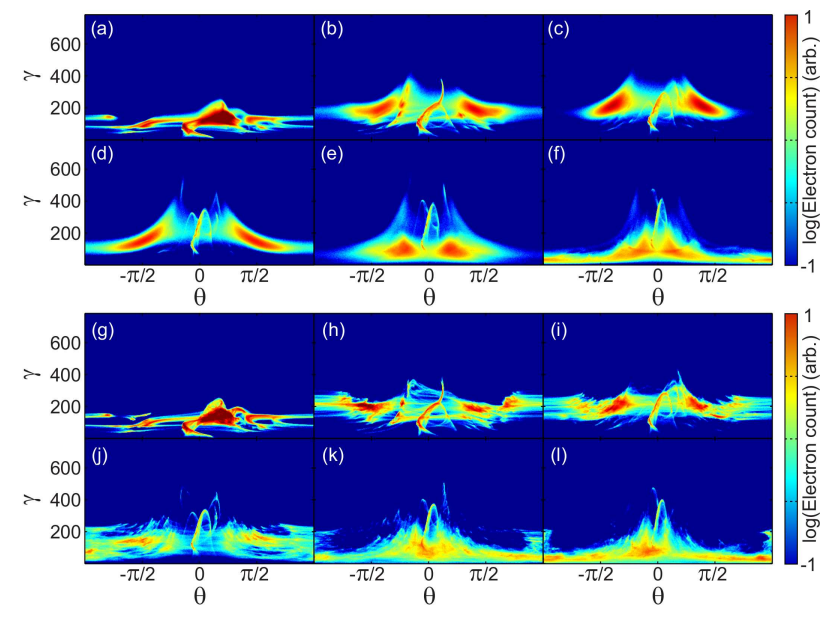

Figure 3: (Color online) Electron relativistic factor $\gamma$ as a function of $\theta$ for $12 T_{L} \leq t \leq 32 T_{L}$, every $4 T_{L}$. (a)-(f) - with RR; (g)-(l) without RR. A transverse window of width $3 \lambda_{L}$ and centered on the laser propagation axis $(y=0)$ is considered.

The RES is composed of two symmetric lobes due to the oscillating nature of the laser field. From figure 1(b) one can estimate initial conditions of the RES:

$$
\begin{aligned}
& \left|\langle\theta\rangle_{t_{0}}\right| \approx \frac{\pi}{2} \\
& \Rightarrow\langle\rho\rangle_{t_{0}} \approx\langle\gamma\rangle_{t_{0}} \approx a_{L}
\end{aligned}
$$

Here, $\langle\ldots\rangle$ defines the average value taken on the electron phase space.

From figure 3 , at $t=16 T_{L}$, the average energy of the $\mathrm{RES}$ is: $\left\langle\mathcal{E}_{e}\right\rangle \approx a_{L} m c^{2} \approx 100 \mathrm{MeV}$ with $|\langle\theta\rangle| \approx \pi / 3$ and its maximum energy $\max \left\langle\mathcal{E}_{e}\right\rangle \approx 1.5 a_{L} m c^{2} \approx 150 \mathrm{MeV}$ with $|\langle\theta\rangle| \approx \pi / 4$. For $t>20 T_{L}$, the electrons within the RES experience a weaker laser intensity. The corresponding electron distribution function expands and the average energy starts drastically decreasing. At $t \geq 30 T_{L}$ the RES no longer experiences the high field, which drastically reduces the RR effect on the electron distribution function and consequently the compactness of the RES. The average angle of the RES is equal to $|\langle\theta\rangle| \simeq \pi / 4$ which implies $\left|u_{x}\right| \approx\left|u_{y}\right|$. By the equipartition theorem it means that this electron population is in thermal equilibrium with an electron temperature $T_{e}$ of the order of $55 \mathrm{MeV}$. Here, the dominant thermalization mechanism is due to the synchrotron radiation emission which tends to decrease the energy of radiating electrons experiencing the laser pulse.

The spectra of high energy synchrotron radiation over time is shown in figures $1(\mathrm{~d}), 1(\mathrm{e}), 1(\mathrm{f})$.

From numerical simulations results, it is stressed that about $80 \%$ of the radiated energy comes from the RES and is emitted over $|\langle\theta\rangle| \approx 0.41 \pi$. We notice that electrons which emit the highest energy photons at the peak of the radiated power $\left(t \approx 18 T_{L}\right)$, satisfy the condition:

$$
\left|u_{z}\right| \approx\left|u_{y}\right| \approx u_{x}>0
$$

which is also confirmed in figure 3 . By contrast, the evolution of the total radiated power for photons generated by forward electrons is uncorrelated to the corresponding electron energy. Since they are accelerated at the front of the laser pulse, they acquire more longitudinal momentum than transverse momentum which results in the average angle tending to 0 over time, as shown in figure 3(a-f). As a result, the average Doppler factor of the forward electrons is greatly reduced, which leads to a decrease of the emission of high energy photons, as shown in figure 1 (f).

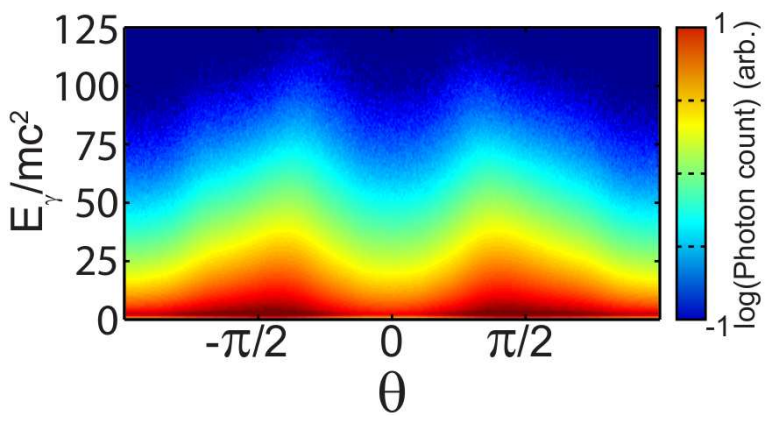

Figure 4: (Color online) Angular distribution of the high energy synchrotron radiation at $t=200 \mathrm{fs}$.

Figure 4 shows the angular distribution of the synchrotron radiation after the laser pulse duration, at $t=$ 
$200 \mathrm{fs}$. The bulk of the high energy photons have an energy of the order of tens of $\mathrm{MeV}$ and can reach a maximum of $\approx 60 \mathrm{MeV}$. The structure of the double lobe characterizing the RES can clearly be seen. This double lobe structure could prove to be a useful signature for the experimental detection of the RES on future $10 \mathrm{PW}$ laser facilities.

The corresponding quantum parameter $\chi_{e}(6)$ of the electrons has also been computed. Almost all electrons have an associated parameter $\chi_{e}$ less than 0.15 as shown in figure 1(g), 1(h), 1(i). Moreover, the highest quantum electrons are forward-directed electrons and those forming the so-called RES have an average $\chi_{e}$ less than 0.1. This insures that the semi-classical treatment is valid.

\section{ANALYTICAL MODEL FOR THE RADIATING ELECTRON SOURCE}

A kinetic model can be derived in order to describe the principal characteristics of the RES. Since the Rayleigh length $L_{R}=\pi w_{0}^{2} / \lambda_{L} \simeq 707 \lambda_{L}$ is much larger than the interaction length $\left(\sim c \tau_{L}=9.1 \lambda_{L}\right)$ on which the study is focused, it is sensible to assume that $a_{L}(x, y, t) \approx$ $a_{L}(y, t)$. As mentioned previously, the simulations focused on a window of width $3 \lambda_{L}$, centered on the laser propagation axis $(y=0)$ in order to highlight that the RES source is localized in the high field areas (see figure 3). Since the laser spot size $w_{0}=15 \lambda_{L}$, this implies $a_{L}(y, t) \approx a_{L}(t)$ for $-1.5 \lambda_{L} \leq y \leq 1.5 \lambda_{L}$. As the areal density of the target $(\xi)$ is [39]: $\xi=\pi \frac{n_{e}}{n_{c}} \frac{l}{\lambda_{L}} \approx 30 \ll a_{L}$, the electrostatic field is therefore much weaker than the laser field. Moreover, due to the ion motion, it follows that the charge separation field is much smaller than it would be with immobile ions. To a first approximation, the charge separation effect on the acceleration of ultrarelativistic electrons can be neglected.

An assumption is therefore made that the laser pulse can be modeled as a plane wave. That is, the electromagnetic field tensor $F^{a}{ }_{b}$ depends uniquely on the phase $\phi:=k^{a} r_{a}=\omega_{L} \int \rho d s$ and is written as:

$$
-\frac{e}{m c^{2}} F_{b}^{a}(\phi)=a_{L} \sum_{i \in\{y, z\}} \psi_{i}(\phi)\left[i^{a} k_{b}-k^{a} i_{b}\right]
$$

where, $k^{a}=\omega_{L}(1,1 / c, 0,0)$ is the null wave vector and $y^{a}=(0,0,1,0)$ and $z^{a}=(0,0,0,1)$ are the polarization vectors. These four vectors satisfy: $k^{2}=k \cdot y=$ $y . z=0$ and $y^{2}=z^{2}=-1$. The functions $\psi_{y}(\phi)$ and $\psi_{z}(\phi)$ describe variations of the electric field strength in the $y$ and $z$ directions, respectively. It is assumed then that the electron distribution function $f_{e}$ depends only on the phase $\phi$ and electron velocity $u^{a}$ such that $f_{e}=f_{e}\left(\phi, \rho, u_{y}, u_{z}\right)$. A closed system for particles is considered, which means that the number of particles is conserved. Neglecting stochasticity quantum effects (see reference [17]), the Vlasov equation takes the form:

$$
\frac{\partial f_{e}}{\partial \phi}+\frac{\partial}{\partial u^{\nu}}\left(f_{e} \frac{d u^{\nu}}{d \phi}\right)=0
$$

Using the equality:

$$
\frac{d}{d \phi}=\frac{\partial}{\partial \phi}+\frac{d u^{\nu}}{d \phi} \frac{\partial}{\partial u^{\nu}},
$$

Eq. (14) can be reformulated as:

$$
\frac{d f_{e}}{d \phi}=-\frac{\partial}{\partial u^{\nu}}\left(\frac{d u^{\nu}}{d \phi}\right) f_{e}
$$

Eq. (16) has a similar form to a Liouville equation, meaning that it is deterministic and tends to decrease the entropy [17]. The right term of Eq. (16) represents the characteristic time of the electron distribution function evolution. The Vlasov equation (16) yields the solution:

$$
f_{e}\left(\phi, \rho, u_{y}, u_{z}\right)=f_{e}\left(\phi_{0}, \rho_{0}, u_{y, 0}, u_{z, 0}\right) e^{\mathcal{G}(\phi, \rho)}
$$

where,

$$
\mathcal{G}(\phi, \rho)=-\int_{\phi_{0}}^{\phi} \mathcal{T}_{c}^{-1}(\varphi, \rho) d \varphi
$$

and

$$
\mathcal{T}_{c}(\phi, \rho):=\left[\partial / \partial u^{\nu}\left(d u^{\nu} / d \phi\right)\right]^{-1} .
$$

The label " $\nu$ " have been introduced for the indices such that $\nu \in\{1,2,3\}$. For readability, the initial variables are written with the index " 0 " instead of the index " $t_{0}$ ". In the following, it is assumed that the initial time corresponds to the formation time $t_{0} \approx 15 T_{L}\left(t_{0} \equiv \phi_{0} / \omega_{L}\right.$ because $\left|\langle\theta\rangle_{t_{0}}\right| \approx \pi / 2$ (11) which implies $k^{\nu} x_{\nu} \approx 0$ ) of the radiating electrons as highlighted in Section IV A. We remind the reader that this corresponds to the time when a significant population of backward-directed electrons are reflected and re-accelerated in the laser field. It can be defined as:

$$
t_{0} \mid \gamma\left(t_{0}\right)=\max \{\gamma|3 \pi / 4 \leq| \theta \mid \leq \pi\} .
$$

Physically, $\mathcal{T}_{c}$ can be interpreted as the kinetic cooling time which is the characteristic time of the evolution of the electron distribution function $f_{e}$. Since the laser pulse duration is finite, the kinetic cooling must depend on the temporal laser profile. This yields:

$$
\frac{d u^{a}}{d \phi}=\frac{d u^{a}}{d s} \frac{d s}{d \phi}=\frac{d u^{a}}{d s} \frac{h\left(\phi, \rho_{0}\right)}{\rho_{0}}=\frac{1}{\rho} \frac{d u^{a}}{d s} .
$$

Using Eq. (5), Eq. (13), $\partial / \partial u^{a}\left[F_{b}^{a} u^{b}\right]=0$ and Eq. (21), the kinetic cooling time can be written:

$$
\mathcal{T}_{c}(\phi, \rho)=\left[4 \omega_{L} \tau_{r} a_{L}^{2} \rho \sum_{i \in\{y, z\}} \psi_{i}(\phi)^{2}\right]^{-1}
$$




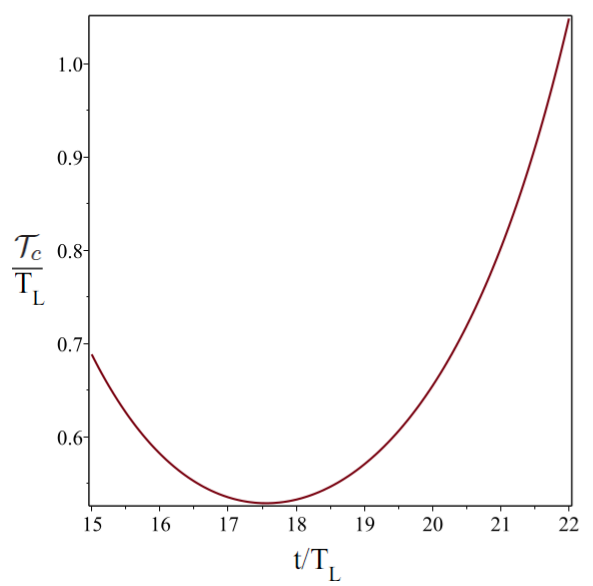

Figure 5: (Color online) cooling time $\mathcal{T}_{c}$ over time.

The evolution of the cooling time $\mathcal{T}_{c}$ is shown in figure 5. It presents a minimum at $t \sim 18 T_{L}$ which corresponds to the moment where the contraction of the electron distribution function of the RES is maximal but also when the radiated power is maximal. This is consistent with the evolution of the angular distribution of the RES shown in figure 3. The cooling time is of the order of one laser period and tends to drastically increase after the laser pulse duration.

Since Eq. (5) has an analytic solution [40], it is possible to find an explicit solution of Eq. (14) by using the method of characteristics and thus expressing the initial phase and velocities $\left\{\phi_{0}, \rho_{0}, u_{y, 0}, u_{z, 0}\right\}$ by independent variables $\left\{\phi, \rho, u_{y}, u_{z}\right\}$ in the following compact form:

$$
\begin{aligned}
& \rho_{0}=\frac{\rho}{h(\phi,-\rho)} \\
& u_{i, 0}=\frac{u_{i}}{h(\phi,-\rho)}-a_{L} c \mathcal{I}_{i}\left(\phi, \frac{\rho}{h(\phi,-\rho)}\right)
\end{aligned}
$$

with,

$$
\begin{aligned}
& \mathcal{I}_{i}\left(\phi, \rho_{0}\right)=\int_{\phi_{0}}^{\phi}\left[h\left(\varphi, \rho_{0}\right) \psi_{i}(\varphi)\right] d \varphi+\omega_{L} \tau_{r} \rho_{0}\left[\psi_{i}(\varphi)\right]_{\phi_{0}}^{\phi} \\
& h\left(\phi, \rho_{0}\right)=1+\omega_{L} \tau_{r} \rho_{0} a_{L}^{2} \int_{\phi_{0}}^{\phi} \psi^{2}(\varphi) d \varphi
\end{aligned}
$$

where,

$$
\begin{aligned}
\psi^{2}(\phi) & =\sum_{i \in\{y, z\}} \psi_{i}^{2}(\phi), \\
\mathcal{I}^{2}(\phi) & =\sum_{i \in\{y, z\}} \mathcal{I}_{i}^{2}(\phi), \\
\psi_{y}(\phi) & =\exp \left[-\frac{\left(\phi-\phi_{*}\right)^{2}}{2 w^{2}}\right] \sin (\phi), \\
\psi_{z}(\phi) & =\exp \left[-\frac{\left(\phi-\phi_{*}\right)^{2}}{2 w^{2}}\right] \cos (\phi) .
\end{aligned}
$$

Here $w=\tau_{L} \omega_{L} / \sqrt{\pi}$ (recall that $\tau_{L}$ is the laser pulse duration) and $\phi_{*}$ denotes the center of the laser pulse field which corresponds to the rise time of the laser pulse. The solutions (23), (24) are consistent with those made by Joffe et al. [19]. When the RR is negligible (formally $\left.\tau_{r} \rightarrow 0\right)$, the function $h\left(\phi, \rho_{0}\right)$ tends to 1 and the electron motion reduces to the standard solution of the Lorentz equation in a plane wave [21]. Using circular polarization prevents strong electron heating by canceling the oscillating component at $2 \omega_{L}$ of the ponderomotive force. This also helps to maintain the compactness of the RES in comparison to linear polarization.

Using (23) it yields:

$$
\mathcal{G}(\phi, \rho)=-4 \ln [h(\phi,-\rho)]
$$

It follows that the electron distribution function can be written as:

$$
f_{e}\left(\phi, \rho, u_{y}, u_{z}\right)=\frac{f_{e}\left(\phi_{0}, \rho_{0}, u_{y, 0}, u_{z, 0}\right)}{h(\phi,-\rho)^{4}}
$$

It is reasonable to assume a Maxwell-Juttner distribution [41] for the initial electron distribution:

$$
f_{e}\left(\phi_{0}, \rho, u_{y}, u_{z}\right)=\mathcal{A} \exp \left[-\frac{(\gamma-1)}{\Theta}\right],
$$

where, $\mathcal{A}:=\frac{n_{e}}{4 \pi c^{3} \Theta K_{2}(1 / \Theta)}, \Theta:=\frac{T_{e}}{m c^{2}}$, and $K_{2}($.$) is the$ modified Bessel function of second order.

The initial temperature of the electron source can be assumed to be close to the ponderomotive scaling such that [42]:

$$
T_{e}\left(\phi_{0}\right) \approx\left(1+a_{L}^{2}\right)^{1 / 2} m c^{2} \approx a_{L} m c^{2}
$$

The relativistic factor of the particle can be expressed as:

$$
\gamma=\frac{\left(1+\rho^{2}\right)+\left(u_{y}^{2}+u_{z}^{2}\right) / c^{2}}{2 \rho},
$$

which yields:

$$
\gamma-1=\frac{(1-\rho)^{2}}{2 \rho}+\frac{u_{y}^{2}}{2 \rho c^{2}}+\frac{u_{z}^{2}}{2 \rho c^{2}}
$$


The electron distribution function can therefore be written in the form:

$$
f_{e}\left(\phi, \rho, u_{y}, u_{z}\right)=\mathcal{A} f_{e, \|}(\phi, \rho) \times f_{e, \perp}\left(\phi, \rho, u_{y}, u_{z}\right)
$$

where,

$$
\begin{aligned}
& f_{e, \|}(\phi, \rho)=\frac{f_{e}\left(\phi_{0}, \rho_{0}\right)}{h(\phi,-\rho)^{2}} \\
& =\frac{1}{h(\phi,-\rho)^{2}} \exp \left[-\frac{\left(1-\frac{\rho}{h(\phi,-\rho)}\right)^{2}}{2 \frac{\rho}{h(\phi,-\rho)} \Theta}\right]
\end{aligned}
$$

is the longitudinal electron distribution function and:

$$
\begin{aligned}
& f_{e, \perp}\left(\phi, \rho, u_{y}, u_{z}\right)=\frac{f_{e}\left(\phi_{0}, \rho_{0}\right)}{h(\phi,-\rho)^{2}} \\
& =\frac{1}{h(\phi,-\rho)^{2}} \exp \left[-\frac{\sum_{i \in\{y, z\}} u_{i, 0}^{2} / c^{2}}{2 \frac{\rho}{h(\phi,-\rho)} \Theta}\right]
\end{aligned}
$$

is the transverse electron distribution function.

By considering the ultra-relativistic regime and electrons included in the RES such that $\pi / 4 \leq|\theta| \leq 3 \pi / 4$, it follows that $\left(1-\rho_{0}\right)^{2} \simeq \rho_{0}^{2}$, which simplifies the expression of the longitudinal electron distribution function $f_{e, \|}(\phi, \rho)$ :

$$
f_{e, \|}(\phi, \rho) \simeq \frac{1}{h(\phi,-\rho)^{2}} \exp \left[-\frac{\rho}{2 \Theta h(\phi,-\rho)}\right]
$$

Basically, the RR tends to decrease (by the function $h(\phi,-\rho))$ the width at half the height of the initial longitudinal electron distribution function leading to a decrease in the average electron temperature which is related to the contraction of the electron phase space [5]. This phenomenon contributes to the generation of the RES by increasing the number of electrons in the highest field areas, as discussed previously in Section IV and shown in figure 3 . However, it is stressed that such a process is no longer valid when quantum stochastic effects become significant since they tend to increase the energy spread [17].

It follows that the decrease of the longitudinal electron temperature can be estimated as:

$$
T_{e}(\phi) \approx T_{e}\left(\phi_{0}\right) h(\phi,-\langle\rho\rangle)<T_{e}\left(\phi_{0}\right)
$$

The evolution of the longitudinal electron temperature is shown in figure 6 .

The situation is different to the case of the transverse electron distribution function. From Eq. (37), it can be noticed that the transverse electron distribution function $f_{e, \perp}\left(\phi, \rho, u_{y}, u_{z}\right)$ depends on $\rho$, which is nothing more than a consequence of special relativity. Therefore, the $\mathrm{RR}$ force does not have the same effect on the transverse dynamics as it has on the longitudinal dynamics. Unlike

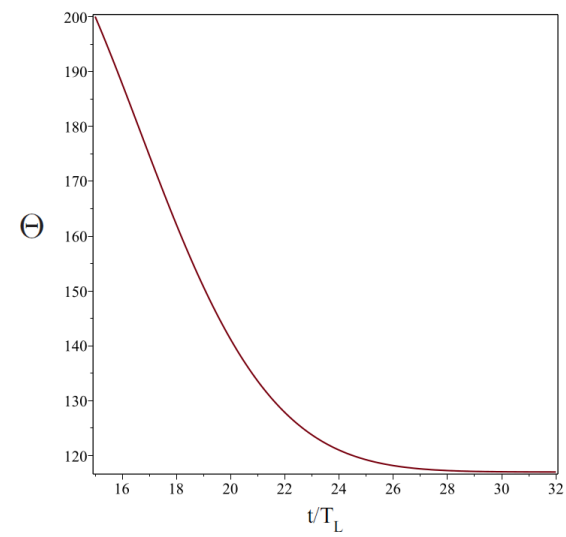

Figure 6: (Color online) Normalized longitudinal temperature $\Theta:=$ $\frac{T_{e}}{m c^{2}}$, over time.

the case of the longitudinal electron distribution function, the RR modifies the shape of the initial transverse distribution. The integral $\mathcal{I}\left(\phi, \frac{\rho}{h(\phi,-\rho)}\right)$ affects the average transverse momentum which causes a shift of $|\langle\theta\rangle|$ to $\pi / 2$ over the laser pulse duration, as shown by comparing figures $3(\mathrm{~b})$ and $3(\mathrm{~h})$.

After the laser pulse duration (formally $\phi \rightarrow \infty$ ), the electron temperature can be explicitly written as:

$$
\begin{array}{r}
T_{e}(\phi \rightarrow \infty) \approx T_{e}\left(\phi_{0}\right) h\left(\phi \rightarrow \infty,-\langle\rho\rangle_{\infty}\right) \\
\quad \approx a_{L}\left[1-\frac{\langle h\rangle_{\infty}-1}{\langle h\rangle_{\infty}}\right] m c^{2} \approx 117 m c^{2}
\end{array}
$$

where,

$$
\langle h\rangle_{\infty}:=h\left(\phi \rightarrow \infty,\left\langle\rho_{0}\right\rangle\right)=1+\omega_{L} \tau_{r}\left\langle\rho_{0}\right\rangle a_{L}^{2} \int_{\phi_{0}}^{\infty} \psi^{2}(\varphi) d \varphi
$$

which is in good agreement with the features of the electron angular distribution observed at $t=200 \mathrm{fs}$, as shown in figure 3(f). Using definition (21) and Eq. (33), the absolute value of the characteristic angle of the RES after the laser field has decayed can be expressed as:

$$
\left|\langle\theta\rangle_{\infty}\right| \approx \arccos \left[\frac{1}{\langle\beta\rangle}\left[\frac{\langle\gamma\rangle-\langle\rho\rangle}{\langle\gamma\rangle}\right]\right]
$$

Using the initial conditions of the RES (11), Eq. (23), Eq. (24) and Eq. 33 it yields:

$$
\left|\langle\theta\rangle_{\infty}\right| \approx \arccos \left[\frac{\langle h\rangle_{\infty}^{2}+a_{L}^{2}}{\langle h\rangle_{\infty}^{2}+3 a_{L}^{2}}\right] \simeq 0.39 \pi .
$$

Note that the RR has a weak influence on the value of the average angle $\langle\theta\rangle$ since $h^{2} \ll a_{L}^{2}$. Also, as noted previously, the most prominent effect of RR is to significantly enhance the electron number in the region for which $\pi / 4 \leq|\theta| \leq 3 \pi / 4$ giving rise to the RES. 


\section{DISCUSSION}

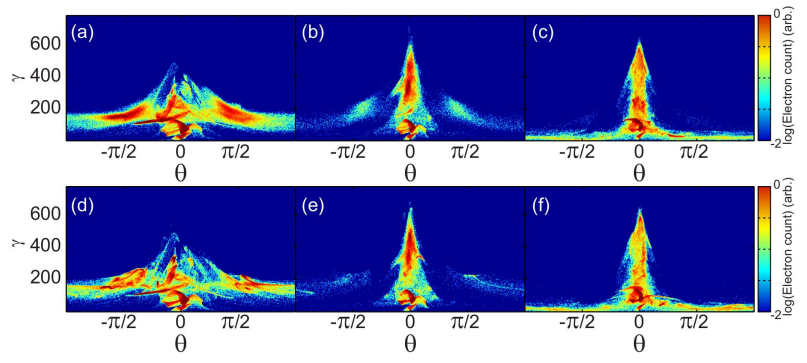

Figure 7: (Color online) Electron relativistic factor $\gamma$ as a function of $\theta$ for $16 T_{L} \leq t \leq 24 T_{L}$, every $4 T_{L}$. (a)-(c) - with RR; (d)-(f) without RR. A transverse window of width $1.7 \lambda_{L}$ and centered on the laser propagation axis $(y=0)$ is considered.

The above numerical analysis was performed with a laser focal spot of $w_{0}=15 \lambda_{L}$ (intensity equal to $1.1 \times 10^{23}$ $\mathrm{W} / \mathrm{cm}^{2}$ ), which enables the 1D kinetic model described in Section V to be derived. We note that the resulting power required to produce this condition is much higher than will be achieved with laser systems in the near future. When the laser focal spot size is reduced to that proposed to produce the required intensity with planned $10 \mathrm{PW}$ systems $\left(w_{0}=1.7 \lambda_{L}\right)$, similar double lobe structures can still be observed, albeit with reduced electron numbers due to the smaller interaction area. We note that some characteristics of the RES, in particular the average angle $\langle\theta\rangle$ and the characteristic energy $\left\langle\mathcal{E}_{e}\right\rangle$ remain unchanged as they depend on the laser intensity. However, we note that the smaller laser focal spot size enhances the transverse ponderomotive force, which tends to reduce the radiation reaction effect, and in addition, the duration of the RES. This is shown in figure 7 for completeness.

In conclusion, due the counteraction of the radiation reaction force on the action of the transverse and longitudinal ponderomotive forces, a compact radiating electron source can be created in the highest field areas, where $\approx 80 \%$ of the high energy synchrotron radiation is emitted over the laser pulse duration. Such a radiating electron source can be produced by the interaction of an ultraintense laser pulse with a near-critical density plasma. The bulk of the synchrotron radiation is of the order of tens of $\mathrm{MeV}$ whilst the highest energy photons can reach energies of $\sim 60 \mathrm{MeV}$. A kinetic model has been derived and confirms characteristics of the radiating electron source such as the evolution of both the longitudinal electron temperature and the electron distribution function through the cooling time. Under these conditions, the classical approach to compute both the electron motion and the radiation emission remains valid. We note that although the physics explored in this paper is highlighted with a large laser spot size, it still remains (albeit less evident) for lower laser spot sizes. The angular distribution as well as the average energy of such a radiating electron source could be potentially detected and measured on future $10 \mathrm{PW}$ laser facilities.

\section{ACKNOWLEDGMENTS}

We acknowledge helpful advice from Dr. A. Noble. This work is supported by EPSRC (grant numbers EP/J003832/1, EP/M018091/1 and $\mathrm{EP} / \mathrm{L} 000237 / 1)$. EPOCH was developed under EPSRC grant EP/G054940/1. Data associated with research published in this paper is accessible at http://dx.doi.org/10.15129/2aadd4e6-2311-4172-afa084a464462aec.

*remi.capdessus@strath.ac.uk

† paul.mckenna@strath.ac.uk

[1] F. Andersson, P. Helander, and L. G. Eriksson, Phys. Plasmas 8, 5221 (2001).

[2] A. Zhidkov, J. Koga, A. Sasaki, and M. Uesaka, Phys. Rev. Lett. 88, 185002 (2002).

[3] A. Di Piazza, K. Z. Hatsagortsyan, and C. H. Keitel, Phys. Rev. Lett. 102, 254802 (2009).

[4] C. Harvey and M. Marklund, Phys. Rev. A 85, 013412 (2012).

[5] M. Tamburini, F. Pegoraro, A. Di Piazza, C. H. Keitel, T. V. Liseykina, and A. Macchi, Nucl. Instrum. Meth. B 653, 181 (2011).

[6] M. Tamburini, T. V. Liseykina, F. Pegoraro, and A. Macchi, Phys. Rev. E 85, 016407 (2012).

[7] T. Schlegel, and V. T. Tikhonchuk, New. J. Phys. 14, 073034 (2012).

[8] A. Di Piazza, C. Mller, K. Z. Hatsagortsyan, and C. H. Keitel, Rev. Mod. Phys. 84, 1177 (2012).

[9] P. Michel, C. B. Schroeder, B. A. Shadwick, E. Esarey, and W. P. Leemans, Phys. Rev. E 74026501 (2006).

[10] G. Lehmann and K. H. Spatschek Phys. Rev. E 84 046409 (2011).

[11] M. Tamburini, C. H. Keitel, and A. Di Piazza, Phys. Rev. E 89, 021201(R) (2014).

[12] D. G. Green and C. N. Harvey, Phys. Rev. Lett 112, 164801 (2014).

[13] L. L. Ji, A. Pukhov, E. N. Nerush, I. Yu. Kostyukov, B. F. Shen, and K. U. Akli, Phys. Plasmas 21, 023109 (2014).

[14] R. Capdessus, E. d'Humiéres, V. Tikhonchuk, Phys. Rev. Lett. 110, 215003 (2013).

[15] R. Capdessus and P. McKenna, Phys. Rev. E 91, 053105 (2015).

[16] E. N. Nerush and I. Y. Kostyukov, Plasma Phys. Control. Fusion 57, 035007 (2015).

[17] N. Neitz and A. Di Piazza, Phys. Rev. Lett. 111, 054802 (2013).

[18] N. Neitz and A. Di Piazza, Phys. Rev. D 90, 022102 (2014).

[19] S. R. Yoffe, Y. Kravets, A. Noble, and D. A. Jaroszynski, New J. Phys. 17, 053025 (2015). 
[20] J. Koga, T.Zh. Esirkepov, S.V. Bulanov, Phys. Plasmas 12, 093106 (2005).

[21] L. D. Landau and E. M. Lifschitz, The Classical Theory of Fields 4th ed. vol. 2 Pergamon New York (1994).

[22] J. Schwinger, Phys. Rev. 82, 664 (1951).

[23] C. P. Ridgers, C. S. Brady, R. Duclous, J. G. Kirk, K. Bennett, T. D. Arber, and A. R. Bell, Phys. Plasmas 20, 056701 (2013).

[24] A. R. Bell and J. G. Kirk, Phys. Rev. Lett. 101, 200403 (2008).

[25] A. I. Nikishov and V. I. Ritus, Sov. Phys. JETP 195 (1964).

[26] A. I. Nikishov and V. I Ritus, Sov. Phys. JETP, 291 (1969).

[27] I. V. Sokolov, N. M. Naumova, J. A. Nees, and G. A. Mourou, Phys. Rev. Lett. 105195005 (2010).

[28] N. V Elkina, A. M. Fedotov, I. Yu. Kostyukov, M. V. Legkov, N. B. Narozhny, E. N. Nerush, and H. Ruhl, Phys. Rev. ST Accel. Beams 14, 054401 (2011).

[29] M. Lobet, E. d'Humiéres, M. Grech, C. Ruyer, X. Davoine, L. Gremillet, J. Phys.: Conf: Ser. 688, 012058 (2016).

[30] J. G. Kirk, A. R. Bell, and C. P. Ridgers, Plasma Phys. Controlled Fusion 51, 085008 (2013).
[31] I. V. Sokolov, N. M. Naumova, and J. A. Nees, Phys. Plasmas 18, 093109 (2011).

[32] T. D. Arber, K. Bennett, C. S. Brady, A. LawrenceDouglas, M. G. Ramsay, N. J. Sircombe, P. Gillies, R. G. Evans, H. Schmitz, A. R. Bell and C. P. Ridgers, Plasma Phys. Control. Fusion 5711 (2015).

[33] T. Erber, Rev. Mod. Phys. 38, 626 (1966).

[34] V. I. Ritus and Tr. Fiz, Inst. Akad. Nauk SSSR 111, 6 (1979).

[35] V. I. Ritus, J. Russ. Laser Res. 6, 497 (1985).

[36] V. Baier and V. M. Katkov, Sov. Phys. JETP 26, 854 (1968).

[37] C. P. Ridgers, J. G. Kirk, R. Duclous, T. G. Blackburn, C. S. Brady, K. Bennett, T. D. Arber, A. R. Bell, J. Comput. Phys. 26027385 (2014).

[38] R. Capdessus, M. Lobet, E. d' Humiéres, V. T. Tikonchuk, Phys. Plasmas 21, 123120 (2014).

[39] A. Macchi, S. Veghini, and F. Pegoraro, Phys. Rev. Lett. 103, 085003 (2009).

[40] A. Di Piazza Lett Math Phys 83, 305313 (2008).

[41] F. Juttner, Annalen der Physik 339, (5) 85688 (1911).

[42] S. C. Wilks, W. L. Kruer, M. Tabak, and A. B. Langdon, Phys. Rev. Lett. 69, 1383 (1992). 